\title{
AUSFÄLLE, BEZIEHUNGEN UND EMOTIONEN
}

„Der Krieg mußte es uns ja bringen, das Große, Starke, Feierliche. Er schien uns männliche Tat, ein fröhliches Schützengefecht auf blumigen, blutbetauten Wiesen“".

Ernst Jünger 1

\section{Noisseville - Antlitz einer Schlacht}

Es soll zunächst vom größten französischen Ausfall, dem zweitägigen Kampf um Noisseville und St. Barbe vom 31. August und 1. September die Rede sein. Neben den Ausfällen gegen Mercy le Haut und Courcelles am 27. September, St. Remy am 2. und Woippy am 7. Oktober war dies der kraftvollste und damit aussichtsreichste Versuch, den deutschen Belagerungsring zu sprengen. Auch kam seinem Erfolg oder Mißerfolg im Zusammenhang mit dem Ausgang der Schlacht bei Sedan kriegsentscheidende Bedeutung zu. Gegenüber allen anderen Scharmützeln des Belagerungskrieges ist Noisseville eine echte Ausfallschlacht. Man will den Durchbruch, strebt so nach dem Absoluten und einer grundsätzlich veränderten Lage ${ }^{2}$. Die günstige Ausgangsposition der Rheinarmee beruhte auf dem militärstrategischen Vorteil der inneren Linie, die es erlaubte, an beiden Schlachttagen eine überlegene Massierung von Menschen und Material auf der Chambière-Insel und im Schatten des Forts St. Julien rechts der Mosel zu erwirken ${ }^{3}$. Zudem war ein motivationales Plus auf seiten der Eingeschlossenen, denn sie fochten ja um die Beseitigung eines für sie unhaltbaren Zustandes und um eine Wende der Kriegslage, während die Deutschen lediglich einen für sie, wie gehört, reichlich unbequemen Status quo aufrecht erhalten mußten.

Zwei hochwichtige Depeschen werden im Vorfeld zwischen den Marschällen Mac-Mahon und Bazaine gewechselt: Zunächst will dieser mit der Heeresgruppe Châlons auf das bedrohte Paris zurückgehen, in der Hoffnung, jener könne sich noch selbst befreien ${ }^{4}$. Doch dann vermeldet Bazaine, daß er die Rheinarmee auf Montmédy und „über die festen Plätze im Norden“ aus dem Kessel herausführen wolle, um sich mit Mac-Mahon zu vereinigen ${ }^{5}$. Dieser entscheidet sich daraufhin auch auf Anraten des Pariser Ministerrats und der Kaiserin, Bazaine entgegenzugehen und damit die Hauptstadt bloß-

1 In Stahlgewittern, S. 7.

2 Vgl. DuBY, Bouvines, S. $122 \mathrm{f}$.

3 Siehe Karte.

${ }^{4}$ Am 19. bzw. 20. August. Vgl. FAY, Tagebuch, S. 66.

5 Ibid. 


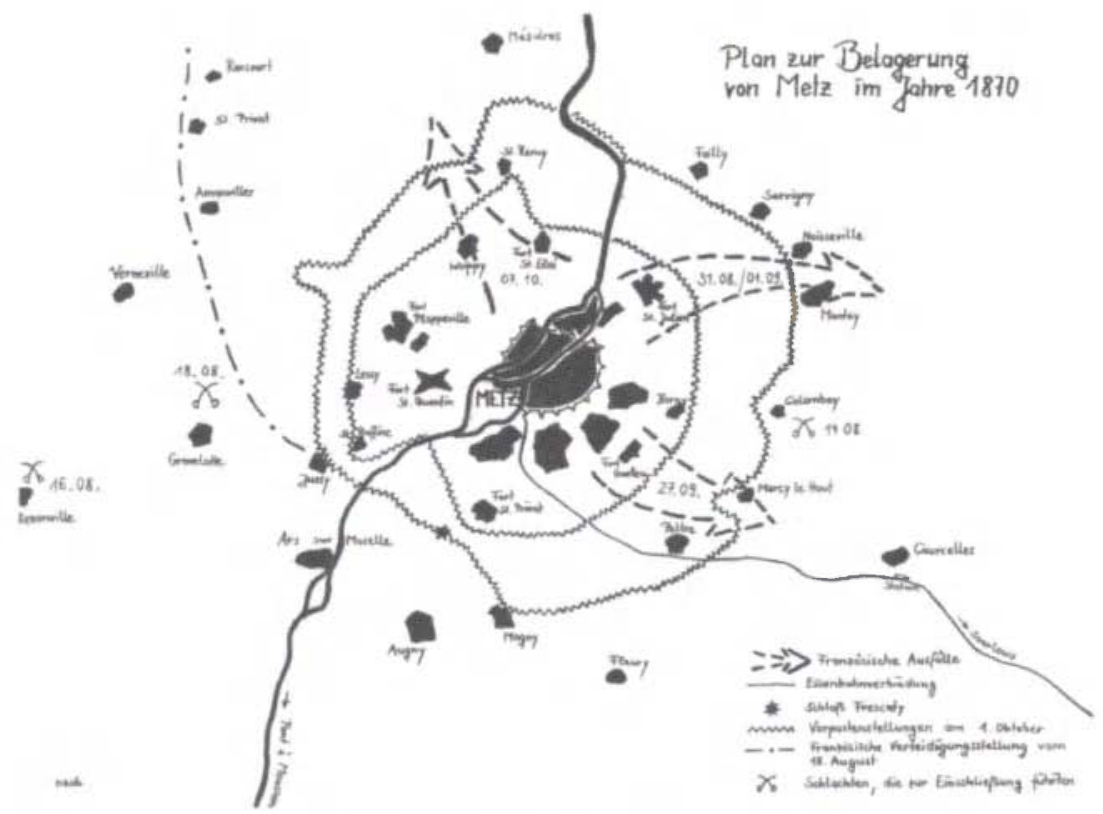

Abb. 12: Plan zur Belagerung von Mctz im Jahre 1870.

zustellen - eine folgenschwere Fehlkalkulation, die in eine tödliche Falle, die „Mausefalle von Sedan“, führt ${ }^{6}$. In Metz aber hofft man an diesem letzten Augusttag auf das Herannahen des berühmten Siegers von Sewastopol und seiner Armee 7 . Das hebt die Kampfmoral.

Daß es den Franzosen ernst war, bewies die sorgfältige Vorbereitung des Unternehmens. Abgesehen von Saarbrücken, gingen sie zum ersten Mal in diesem Krieg in die Offensive. Die Korpsbatterien erhielten ausreichend Artilleriemunition, die Soldaten einen auf drei Schlachttage berechneten eisernen Mundvorrat. Fouragierungsgespanne, beladen mit Speck, Zwieback und Hafer, verließen an beiden Schlachttagen im Schatten der Truppen die Festung ${ }^{8}$. Alle späteren Ausfälle wurden „mit einem Biscuit in der Tasche ${ }^{\text {" }}$ geführt, denn es handelte sich dabei nur noch um großangelegte Fouragierungsunternehmen, die lediglich beim Uberfall auf Peltre am 27. September einhundert Gefangene und fünfzig Ochsen einbrachten ${ }^{10}$. Nachdem der

${ }^{6}$ Als strategischen Fehler herausgestellt auch bei STÄHLIN, Krieg 1870/71. DELBRÜCK, Kriegskunst. Helmert, UsCzecK, Preußisch-deutsche Kriēgé.

7 KüRSCHNER, Krieg, S. 606.

8 GolTz, Feldzug, S. 220 f.

9 RiNifleisCH, Feldbriefe, S. 63.

10 Hanneken, Krieg um Metz, S. 29. Dazu Bazaines Armeebefehl Ende September im Anhang, Anlage V. 
Morgennebel des 31. August gefallen war, konstatierte man deutscherseits große Truppenbewegungen in Richtung der Insel Chambière und des Forts Julien. Von dort aus wollte Bazaine in zwei Angriffskeilen nach Norden und Nordosten auf Diedenhofen durchbrechen ${ }^{11}$. Da die meisten französischen Korps, ebenso wie die deutschen, auf dem linken Moselufer lagerten, dauerte der Aufmarsch der Rheinarmee jedoch bis in die Mittagsstunden. Jetzt erst, zu spät, um den strategischen Erfolg noch am selben Tag erzielen zu können, kam die Offensive in Gang. Die Truppenkommandeure hielten noch einmal flammende Reden, und die Marseillaise wurde angestimmt. Der Hauptstoß des französischen Angriffs zielte gegen Servigny, von wo deutsche Artillerie die Moselstraße beherrschte, sowie gegen Noisseville, das am Abend genommen wurde. Hier entstand eine für die Verteidiger kritische Lage, als die Franzosen bis Retonfay vorgedrungen waren und die zurückgeworfenen Preußen in ihrer linken Flanke umfaßt zu werden drohten. Durch die hereinbrechende Dunkelheit blieb die Schlacht jedoch in der Schwebe. Ein zweiter, nach Norden gerichteter Angriffsstoß traf auf die weit ausgedehnten Linien der 3. Reservedivision unter dem Kommando Generalleutnants von Kummer, die den Frontabschnitt zwischen Malroy und Bois de Failly zu decken hatte. Sie sollte als die "Leidensdivision“ aus den Kämpfen hervorgehen, da sie an beiden Schlachttagen immer mit überlegenen französische Kräften zu tun und die weitaus größten Verluste zu beklagen hatte. Überall war es zum Nahkampf gekommen, der auch nachts immer wieder aufflammte. In einer Depesche vom 1. September $1 \mathrm{Uhr} 40$ hieß es:

Die Franzosen haben nächtlichen Überfall auf Failly-Servigny-Noisseville gemacht. Ostpreußische Kolben und Bajonette haben gesiegt. Wir halten die Position $[\ldots]^{12}$

Am Morgen des 1. September erneuerten die Franzosen ihre Angriffe. Entlang der Straße nach Saarlouis rückte Einheit um Einheit vor. Während die deutschen Feldtelegraphen heißliefen, um alle disponiblen Verstärkungen in Richtung der bedrohten Frontabschnitte zu dirigieren, prallten dort die beiderseitigen Infanteriemassen mit äußerster Heftigkeit aufeinander. Es entwickelte sich ein hin- und herwogendes Feuergefecht, das die Franzosen lange Zeit erfolgreich in Noisseville und Flanville sah. Von dort trugen sie ihre Angriffe vor. Immer wieder ließen sie ihre Tirailleurs ausschwärmen, um die Deutschen aus ihren Stellungen zu werfen. Ohrenbetäubender Lärm narkotisierte die Sinne der Kämpfenden, die sich, umschwirrt von Kugeln und umgeben von Pulverdampf, geduckt lauernd vorwärts bewegten. Mut und

$11 \mathrm{Ob}$ Bazaine lediglich alle Streitkräfte der Deutschen vor Metz binden wollte, um Mac-Mahon den Rücken frei zu halten, wie Delbrück es behauptet hat, oder ob er wirklich mit Mann und Maus durchbrechen wollte, läßt sich mit Gewißheit nicht sagen. Jedoch wäre ein Durchbruch und damit ein Sieg der Franzosen bei Noisseville längerfristig durchaus ein Gegengewicht zur Niederlage bei Sedan gewesen: DELBRÜCK, Geschichte der Kriegskunst, S. $334 \mathrm{f}$.

12 GolTz, Feldzug, S. 214 
Angst trieben sie an. Unter extremer Anspannung erwartete man den Zusammenstoß mit dem Gegner. Dabei waren die angreifenden Franzosen psychologisch im Vorteil, da sie sich durch eine stärkere motorische Aktivität ablenken konnten ${ }^{13}$. Die Pulse flogen hier wie dort.

Was aber passiert nun wirklich? Viele Soldaten beten vor oder während der Angriffe. Andere reißen sich aus Aberglauben die Erkennungsmarken von der Brust oder werfen die Spielkarten weg, weil sie fürchten, diese würden feindliche Kugeln anziehen ${ }^{14}$. Auch beflügelt der Wille, vor den Kameraden nicht zu versagen oder Vorgesetzten zu imponieren. Im Augenblick des Kampfes funktionieren die um Selbstschutz und Aggression kreisenden Instinkte, die im Nahkampf zu Tötung oder Tod führen. Anfangs wird noch geschossen, dann kommt es zum Kolben- und Bajonettkampf, zuletzt zum Zweikampf mit dem Spaten oder schließlich mit den bloßen Fäusten. Nicht zielgerichtete Handlungen, sondern Reflexe sind jetzt noch bestimmend: Abdrücken, Zustechen und Zuschlagen, Abducken und Zusammenkrümmen; all dieses geschieht fast unbewußt, von den wenigen im Kasernendrill eingeschliffenen Automatismen abgesehen:

[...] man ist halb wie im Traume, die Eindrücke von Außen wirken unklar und nur in ihren stärksten Erscheinungen. Manche sind leichenfahl oder quittegelb, von Kälte durchrieselt, andere vor Hitze und Aufregung puderrot, mit triefendem Schweiß auf der Stirn. Dem einen flimmert es vor Augen, dem anderen füllen sie sich mit Thränen, oder sie sind ihm wie ausgetrocknet. Einer verfällt geradezu in Weinkrämpfe. Der Mund ist halb geöffnet, die Lippen sind nach innen gekniffen oder aufgeworfen, die Kehle ist wie zugeschnürrt, als würde man erdrosselt, die Zunge klebt trocken am Gaumen, im Munde ein fauler erdiger Geschmack, im Magen ein Gefühl von Ekel und Übelkeit. ${ }^{15}$

Hier verliert der Krieg jede Romantik, und alles Heroische wird bedeutungslos. Es bleibt nur mehr der nackte Existenzkampf.

Am späten Vormittag änderte sich die Situation. Veranlaßt durch das Eintreffen starker Artilleriereserven ließ der kommandierende General des I. Armeekorps die Infanterie zurücknehmen und ein vernichtendes Feuer auf die französischen Stellungen eröffnen. Allein 78 Kanonen richteten ihre Rohre auf Noisseville und die naheliegende Brasserie L' Amitié, und wie so oft in diesem Krieg entschied auch hier wieder die Überlegenheit der deutschen Artillerie über Sieg und Niederlage ${ }^{16}$. Dem schweren Beschuß vermochten die Franzosen nichts mehr entgegenzusetzen. Sie räumten ihre gewonnenen Positionen und wichen langsam unter die Festungswerke ins Moseltal zurück. Gegenseitige Schuldzuweisungen der Korpskommandanten folgten und wurden Jahre später im Prozeß gegen Bazaine wieder aufgewärmt ${ }^{17}$.

13 Kühlich, Die Soldaten, S. 379f. Pfi.Ugk-HartTung, Schlacht, S. 14-16.

$14 \mathrm{KoCH}$, Bei den Fahnen, S. 65.

15 Pflugk-HartTung, Schlacht, S. 20. Zur individuellen Erfahrung des Nahkampfes immer noch am ehesten authentisch: JÜNGF.R, In Stahlgewittern.

16 Der Deutsch-Französische Krieg 1870-71, Bd. II, S. 924.

17 BAZAINe, Episoden, S. 64-67. 
Dem Kriegsberichterstatter Engels ist es nach der Kapitulation gänzlich unbegreiflich, wie sechzehn Divisionen ausgezeichneter französischer Infanterie von drei feindlichen Landwehrdivisionen zurückgetrieben werden konnten $^{18}$. Man vermutet Sabotage oder eben eine bereits stark fortgeschrittene Demoralisierung der Truppe.

Um 12 Uhr schwiegen nach fast dreißigstündigem Kampf und dem völligem Scheitern aller französischen Durchbruchsversuche die Waffen. Auf französischer Seite hatten nach und nach 120000, auf deutscher etwa 70000 Mann an der Schlacht teilgenommen. Wie später in den Stellungskämpfen des ersten Weltkrieges, zeigte sich, daß zahlenmäßig unterlegene Einheiten in gut verschanzten Positionen auch gegen eine große Übermacht von Angreifern standzuhalten vermochten. Die beiderseitigen Verluste waren beträchtlich: So meldete die deutsche Seite 2990, die französische 3547 Mann tot, verwundet oder vermißt ${ }^{19}$. Am Nachmittag telegraphierte Bazaine an den Kriegsminister:

Nach einem mit aller Kraft ausgeführten Vorstoß [...], befinden wir uns wieder im befestigten Lager von Metz, mit wenigen Hilfsquellen für Munition zur Feldartillerie, Fleisch, Schiffszwieback [...]. Ich fahre fort, Anstrengungen zu machen, um aus der Lage, in welcher wir uns befinden, herauszukommen $[\ldots]^{20}$

Da zur selben Zeit Sedan kapitulierte, Frankreich seinen Kaiser und MacMahon, seinen besten General samt 100000 Mann verlor ${ }^{21}$, sank diese Hoffnung unter den Eingeschlossenen in der Folgezeit auf ein Minimum herab. Daran vermochte auch die patriotische Aufbruchstimmung nichts mehr zu ändern, die nach Bekanntwerden der republikanische Wende in Paris Teile der Bevölkerung ergriff. Das Militär blieb davon, wie erwähnt, im wesentlichen unbeeindruckt. Auf beiden Seiten erkannte man nun langsam an, daß die Rheinarmee an Metz gekettet bleiben würde und lediglich noch als ein immobiler Machtfaktor im Krieg, möglicherweise auch darüber hinaus anzusehen war.

\section{Franctireurs und Feindbilder}

Vor allem die Zwangseinquartierungen und Requirierungen, die den Bewohnern des Umlandes im Zuge des Belagerungskrieges auferlegt wurden, sorgten für einen wachsenden Widerstandswillen innerhalb der französischen Bevölkerung. Da die Beschlagnahmung von Privatbesitz dezentral, also auf $\mathrm{Ba}-$ taillons- oder gar Kompanieebene organisiert und vollzogen wurde, mußten viele Dörfer Kommandos dieser Art mehrfach über sich ergehen lassen. Was

18 ENGELS, Über den Krieg - XXVI, in: MEW, S. 158-160, hier S. 160.

19 Bazaine, L' Armée, S. 214 f. Paulus, Cernirung, S. 70.

20 KÜRSCHNER, Krieg, S. 612.

21 Ausführlich dazu FidelaK, Sedan, S. $42 \mathrm{f}$. 
die einen nicht nahmen, beschlagnahmten die anderen. Ställe, Keller und Kisten mußten geöffnet werden, trotz allem Flehen und Klagen. „Die armen Leute erhalten nur einen Schein für das Genommene mit dem Bemerken: Napoleon bezahlt alles!!!“22 Die vollständige Ruinierung der Zivilbevölkerung war die zwingende Folge einer solchen Kriegsführung. Paradoxerweise sah sich nun wiederum das deutsche Oberkommando in die Pflicht genommen, die Betroffenen aus Heeresbeständen notzuversorgen ${ }^{23}$. Die Einheimischen hingegen empfanden es als entwürdigend, gerade von jenen das Gnadenbrot zu empfangen, die ihr eigenes Unglück verursacht hatten. Mancher deutsche Soldat registrierte dies, fühlte sich mitschuldig und litt auch seelisch darunter:

Der ohnmächtige Kampf der Aermsten gegen den Gewaltigen, der bald höflich bittet, bald überredet, bald leiht, bald zuredet und verspricht - und schließlich doch mit der drohenden Faust nehmen muß! - Es war mir im Anfange furchtbar, aber man muß über alle diese Windungen, in denen sich das Interesse des Einzelnen hindurchhelfen möchte, mit dem todten Zwange hinweggehen, und man gewöhnt sich zuletzt daran, weil man ja selbst einen Kampf um das Unentbehrlichste kämpft. ${ }^{24}$

Angesichts dieser Härten nahm es nicht Wunder, daß eine steigende Zahl vor allem jüngerer Männer den sogenannten Franctireurs beitraten. Diese bewaffneten Zivilisten führten eine Art Partisanenkampf gegen die Deutschen, der oft als Volkskrieg bezeichnet wurde. Die französische Presse hatte die $\mathrm{Pa}$ role dazu ausgegeben und in blutrünstig-drohenden Tönen die totale Erhebung gegen die Eindringlinge beschworen ${ }^{25}$. Gerade die belagerten Plätze und deren Umland waren davon betroffen, und die Ereignisse dort boten viel Zündstoff. Unerfahren im Partisanenkrieg und ebensowenig überzeugend in der Rechtfertigung von Gewalt gegen eine kämpfende Zivilbevölkerung, lief das deutsche Militär der französischen Propaganda so „ins offene Messer"26, noch ehe es tatsächlich zu größeren Strafexpeditionen, wie im späteren Loire-Feldzug, gekommen war, wo in den bunt zusammengewürfelten republikanischen Haufen die Unterschiede zwischen Mobil- und Nationalgarden sowie den Franctireurs immer mehr verwischten. Hier zeigte das Franctireurwesen dann auch Wirkung, vornehmlich durch ständiges Stören des deutschen Vormarsches sowie durch Sabotage rückwärtiger Verbindungen, besonders der Bahnlinien. Was die Belagerungszeit anbetraf, so fand sich in französischen Blättern nun der preußische Ulane, wie er mit gezücktem Messer eine Metzer Bäuerin überfiel, Greise und Kinder tötete, Nonnen die Hände abschnitt ${ }^{27}$, womit der $\mathrm{Haß}$ gegen einen plündernden, vergewaltigen-

22 LEGEWITT, Feldpostbriefe, S. 19.

${ }^{23}$ KRETSCHMAN, Kriegsbriefe, S. 108.

24 RindfleisCH, Feldbriefe, S. 37.

25 JEISMANN, Vaterland, S. 208-211.

26 Ibid S. 291.

27 Gobineau verfolgte die Geschichte, die sich im Elsaß zugetragen haben soll und ihren Weg bis ins Département Oise nahm, rückwärts: aus der Nonne wurde eine Wirtin, 
den und mordenden Feind geschürt war ${ }^{28}$. Andererseits sprach man von "entmenschten Bauern“, die in „hunnenhafter Wut" deutsche Soldaten hinterrüicks ermordeten, und bemühte sich, das Bild vom guten Besatzer, der ein französisches Kind im Arm hielt, zu zeichnen. Doch so wenig letzteres die Franzosen hätte dazu bewegen können, die Deutschen in Lothringen als Befreier zu akzeptieren, so wenig führten die wüsten Kampagnen etwa des Petit Journal - einer Art französischer Bild-Zeitung von damals - zum gewünschten Effekt. Von deutscher Seite beschimpfte man die Franzosen als „Hunde“, "Gesindel“ und „Halunken“, diffamierte sie auch als "Hanswurste in roten Hosen“ oder als „Nußknacker"29. Obwohl immer wieder vom „verkommenen Volk" die Rede war, lag dem, sieht man einmal von den noch zu behandelnden geschlechtertypischen Zuschreibungen ab, ein konkretes, auf die Nation gemünztes Feinbild kaum zu Grunde. Auf französischer Seite höhnte man öfter über die preußischen "Krautjunker“, war jedoch anders als in Deutschland bemüht, den aus den Befreiungskriegen herrührenden Topos nationaler Erbfeindschaft durch einen tiefer liegenden Gegensatz von Zivilisation und Barbarei zu verschärfen. Die Deutschen mußten so - eben und gerade wie vor Metz - als „Barbaren“, „Vandalen“ oder auch als „hordes prusiennes" erscheinen, wohingegen sich Frankreich zum Verteidiger der Hochkultur erklärte ${ }^{30}$. Die deutschen Ideen von 1914, die zur Dämonisierung des Feindes dann "machtgeschützte Innerlichkeit“ (Thomas Mann) gegen oberflächliche Zivilisation, organische Gemeinschaft gegen mechanische Gesellschaft, Helden gegen Händler stellten ${ }^{31}$, sind so gesehen nur eine Umkehrung dessen, also letztlich ein Reflex und die Nachahmung französischer Vorbilder.

Im Metzer Umland hatten sich nach dem Sturz des Kaisertums und unter dem steigenden Druck der Besatzer die Freischärlergruppen gemehrt:

Diese feindlichen Detachements werden von Förstern und Zollbeamten geführt, die Bauern machen die Spione und Wegweiser. So schleichen sie sich in der Nacht durch die Wälder an die Dörfer und Wachen heran und schießen mit Schrot hinein, gegen allen Kriegsgebrauch. Der Krieg nimmt durch solche Infamie einen erbitterten Charakter an [...]. Repressalien können nicht ausbleiben, und das Land wird schließlich die Zeche bezahlen, denn so gutmütig auch unsere Leute sind, so muß doch mit Energie zu ihrem

dann eine Wirtin, die noch ihre Hände hatte, und bei der zuletzt sächsische Soldaten Branntwein getrunken und ordnungsgemäß bezahlt hatten. Zit. nach BRONNER, 1870/ 71, Bd. 1, S. 201 und Bd. 2, S. 457.

28 Ibid S. 212-214. Vgl. auch die Abb. „Le Hulan et la Paysanne“ bei Jeismann, Vaterland, S. 213.

29 KÜHLICH, Soldaten, S. 147-150.

30 JeISMANN betont die Nähe zur Argumentation von 1792. DeRS., Vaterland, S. 207 234.

31 Vgl. hierzu u. a. Werner SOMBART, Händler und Helden. Patriotische Besinnungen, München 1915. Thomas MANN, Betrachtungen eines Unpolitischen, München 1918. 
Schutze vorgegangen werden, und Totschießen und Niederbrennen der Ortschaften wird in nächster Zeit an der Tagesordnung sein. ${ }^{32}$

Zum Teil sind solche Trupps besoldet und militärisch instruiert ${ }^{33}$, zum Teil handeln sie auf eigene Faust. Wie oben herausgestellt, benutzen sie unerlaubte, „unedle“ Waffen und sind auch sonst wegen ihrer hinterhältigen, mordbrennerischen Kriegsführung berüchtigt. Das erinnert an mittelalterliche Söldner jener Gegenden, die sogenannten „cottereaux“, die neben den Rittern nicht mit dem Schwert, sondern mit dem Messer (couteau), eben unedel und unehrlich kämpften ${ }^{34}$. Man spricht auch von „Trunkenbolden, Wilddieben, ehemaligen Sträflingen", von Leuten also, die nichts zu verlieren haben ${ }^{35}$. Traurige Berühmtheit erlangen nächtliche Brandstiftungen von Hausbesitzern, die den Besatzern gelten, aber auch die eigenen Familien nicht schonen. In der Regel droht aufgegriffenen Freischärlern die Todesstrafe, in seltenen Fällen die Deportation zur Zwangsarbeit nach Deutschland ${ }^{36}$. Plünderungen und Ausschreitungen deutscher Soldaten gegen Zivilisten - berüchtigtes Zeugnis lieferte die Photographie eines während der Kämpfe im Osten angeblich bei lebendigem Leibe verbrannten Franctireurs $^{37}$ - sind, wie für Bazeilles, verbürgt, bleiben aber noch die Ausnahme. Vergeltungsmaßnahmen an Unschuldigen, ähnlich der bestialischen Exzesse deutscher Truppen im Zweiten Weltkrieg, deuten sich nur leise an, doch senkten sich schon damals Schatten über ansonsten makellos erscheinende Einheiten. Vereinzelt ist von Geiselerschießungen in einem Verhältnis von 1:4 die Rede ${ }^{38}$. Nach einem nächtlichen Überfall am 22. September in Briey, nördlich der Schlachtfelder, bei dem mehrere Preußen den Tod finden, werden neun Freischärler hingerichtet, darunter der Maire und der Schulmeister, weil sie den Aufenthalt preußischer Truppen im Ort angezeigt hatten ${ }^{39}$. Mit dem Fall des Kaiserreichs, nachdem also die regulären Armeen Frankreichs verschwunden sind, mehren sich die Berichte von militärischen Exekutionen gegen offenbar deutlich renitenter werdende Zivilisten. Man berichtet mit ei-

32 Briefe des Generals der Infanterie von Vorgts-RHETZ, S. 150 f. Ähnlich: KRETSCHMAN, Kriegsbriefe, S. 92.

33 In Metz als halboffizielle Truppe seit Mitte Juli formiert, aus Beständen der Garnison bewaffnet und mit 3 F. pro Tag gut versorgt. Vgl. DENIS, Garnison, S. 203 f.

34 DuBY, Bouvines, S. 85.

35 Gobineau, Ce qui est arrivé, S. 183.

36 Siehe Anhang/Anlage I.

37 Auf Chateau de Pouilly bei Dijon. Abbildung bei JEISMANn, Vaterland, S. 226f. Tatsächlich war der Mann zuvor von eigenen Leuten erschossen worden. KÜRSCHNER, Krieg, S. 1127-1130.

38 So angedroht vom Kommandanten der Festung Laon nach deren Sprengung durch Sabotage. Vgl. VS (19. Oktober 1870).

${ }^{39}$ HirTh, Tagebuch, Bd. II, S. 2353 f. Hierher das Strafgericht gegen Albis, wo am 7. Oktober preußische Husaren im Schlaf niedergemacht worden waren. Wie auch in anderen Fällen, wurden alle Häuser des Ortes niedergebrannt und die Bewohner hingerichtet. Vgl. KRETSCHMANS Schilderung der Ausschreitungen in Sens, südöstlich von Paris. Kriegsbriefe, S. 413-430. 
ner gewissen Selbstverständlichkeit von einem durchaus erbarmungslosen Vorgehen gemäß den Festlegungen des Oberkommandos ${ }^{40}$. „Wo immer die deutschen fliegenden Kolonnen", so liest man mit unguten Assoziationen Engels' Kommentar zur Sache, ,in das Herz Frankreichs einmarschieren, ist ihr Weg allzu oft mit Feuer und Blut gezeichnet" ${ }^{\text {"41. }}$.

Unter der französischen Landbevölkerung kursiert hingegen bald das Wort vom prussiens Français, das auf wachsende Schwierigkeiten der Einheimischen mit solchen Mobilgarden und Franctireurs hindeutet, die in "preuBischer" Manier private Vorräte beschlagnahmen, weil sie sich als Schutzherren dazu ermächtigt fühlen ${ }^{42}$. Zivilisten konnten so nicht nur materiell, sondern bereits existentiell in eine fatale $Z_{\text {wickmühle geraten: Bei neutralem }}$ Verhalten von den eigenen Leuten schikaniert, drohte ihnen im Falle der Teilnahme an einem wie auch immer aufgenötigten Widerstand das Strafgericht der Deutschen. Freilich nahm dies noch nicht jene äußersten Formen an, wonach man beim Aushängen einer weißen Fahne Standrecht und Todesstrafe zu fürchten hatte. Im ganzen gesehen, ging von der Franctireurs-Bewegung, die später in der französischen Resistance als F.T.P. - der kommunistischen Partei nahestehenden Francs-Tireurs Partisans - noch einmal eine gewisse Rolle spielen sollte ${ }^{43}$, nicht jener geschlossene Widerstand aus, den man erhofft hatte. Zu einer patriotisch motivierten "guerre à outrance" jedenfalls ist der Volkskrieg trotz der Rache heischenden Aufrufe eines Victor Hugo nirgends geworden. Vielmehr handelte es sich um Erhebungen zunächst spontaner, später koordinierter Art, deren entscheidender Antrieb das persönliche Betroffensein vom Krieg und seinen Auswirkungen, von Requirierungen, Einquartierungen oder familiären Verlusten blieb. Dies war keineswegs neu, denn Besatzungsmächte hatten bereits in früheren Kriegen den Widerstand der unterworfenen Zivilbevölkerung zu spüren bekommen. Ein Novum allerdings bedeutete die Propagandistische Untermauerung der Dinge, und so wurde später trotz der oben angeführten Beispiele wohl zu Unrecht behauptet, erst eine besonders drakonische Besatzungspolitik der Deutschen habe den Widerstand provoziert ${ }^{44}$. Sowenig der zum Mythos gewordene französi-
40 Siehe Anhang/Anlagen I und II.
41 ENGELS, Der Kampf in Frankreich, in: MEW, Bd. 17, S. 167-171, hier S. 169.
42 Kretschman, Kriegsbriefe, S. 204. Hassel, Armee, S. 296 f.
43 Hier neben der Widerstandsbewegung um General de Gaulle. Vgl. PARISSE, Lothrin- gen, S. 469.
44 Ausführlich diskutiert bei KüHLICH, der verschiedene Formen und Motive betont. DERS., Soldaten, S. 303-320. Nicht unerheblich dafür, daß individuelles Betroffensein zu einer patriotisch-nationalen Erhebung, so wie etwa 1813, gerinnt, ist fraglos auch die Dauer der Besatzung eines Landes durch fremde Mächte oder des Aufenthalts feindli- cher Truppen in einer bestimmten Gegend. Ähnliches gilt später für längere Bomben- kriege, die das Solidaritäts- und Zusammengehörigkeitsgefühl unter den Betroffenen nur stärken und nicht lähmen. Die Tatsache, daß der Aufenthalt der Deutschen in Frankreich 1870/71 vergleichsweise kurz war und sich ein allgemeiner Widerstands- wille noch am ehesten unter der Bevölkerung belagerter Plätze regte, steht ebenfalls ge- gen die These eines prinzipiell national motivierten Volkskrieges gegen die Deutschen. 
sche Volkskrieg wirklich ein Volkskrieg war, so stark gehörte hingegen die Erfahrung rüder Partisanenbehandlung zum prägenden Erinnerungshaushalt des wilhelminischen Deutschland. Metz kam hier neben dem erbittert geführten Winterfeldzug an der Loire eine Schlüsselrolle zu. Norbert Elias führt aus Walter Bloems weit verbreitetem Unterhaltungsroman Volk wider Volk (erschienen 1912) eine Episode der Partisanenbekämpfung von 1870/71 an, wonach der Feind zuerst gejagt, sodann gefangen und schließlich mißhandelt wurde - zuletzt nichts anderes mehr war „als eine wilde, tückische Bestie“, die es zu vernichten galt. Die hierin akzeptierte Brutalität „als Zeichen eines grundsätzlich bejahten und sogar lobenswerten Verhaltenskanons" 45 scheint sich mit wachsendem Abstand zum Ereignis im gesellschaftlichen Wertekosmos des Kaiserreichs immer fester verankert zu haben. Paßgerecht dazu kehrten in der Presse beständig jene Schauergeschichten wieder, die von vermeintlichen französischen Greueltaten an deutschen Soldaten berichteten ${ }^{46}$.

\section{Weiblicher Patriotismus und „liederliche Frauenzimmer“}

In der belagerten Stadt begann man sich unter der Last der Belagerung und dem Druck der fremden Heere nun auch an Jeanne d'Arc und die Heldinnen von Beauvais zu erinnern, an Frauen also, die in der französischen Nationalgeschichte eine besondere Rolle gespielt hatten ${ }^{47}$. So appellierte eine Metzer Arbeiterin an ihre Kolleginnen, daß man, deren Beispiel folgend, nun unbedingten Widerstand gegen die bevorstehende Kapitulation der Festung leisten solle. Ihre den Deutschen geltenden Drohungen erinnern an den „weiblichen Heroismus" der Befreiungskriege, an Eleonore Prochaska und andere "Heldenjungfrauen“48. Auch rufen sie uns Tacitus' Schilderungen germanischer Frauen ins Gedächtnis. Denn wie jene im Kampf gegen die Römer ${ }^{49}$, so wollten nun die Frauen von Metz im Krieg gegen die Deutschen als unüberwindliches menschliches Bollwerk hinter ihre Männer treten:

Unstrittig ist aber, daß der französische „Volkskrieg“ durch die republikanische Wende Anfang September einen gewissen nationalen Impuls erfuhr.

45 ELIAS, Studien, S. 237 f. Natürlich gab es kritische Gegenpositionen, die ebenfalls weiteste Verbreitung fanden: Franz Adam BEyerLeIN, Jena oder Sedan, Berlin 1903.

46 Etwa findet sich in der Jenaischen Zeitung vom 30. April 1891 ein Bericht, wonach man beim Niederreißen eines Hauses in Deutsch-Redingen in Lothringen die Leichen zweier 1870 heimlich erschlagener und anschließend eingemauerter deutscher Soldaten gefunden habe.

47 Gemeint waren neben der Jungfrau von Orléans, die den Engländern entgegengetreten war, die Frauen um Jeanne Hachette, die Beauvais im Jahre 1472 erfolgreich gegen Karl den Kühnen verteidigt hatten.

$48 \mathrm{Vgl}$. HagemanN, Heldenmütter, S. $196 \mathrm{f}$.

49 Tacitus, Germania, 7-8. 
Erst nachdem ihr unsere Brüste durchbohrt habt, werdet ihr über unserer und unserer Kinder Asche einherschreitend, den Boden unserer Vaterstadt besudeln können. [...] unsere Hände, sonst nur gewöhnt, eine Nähnadel zu handhaben und Verwundete zu verbinden, können auch, wenn es sein muß, eine Waffe führen. Die nationale Ehre über alles! Lieber den Tod als die Knechtschaft! 50

Angesichts tödlicher Gefahr trat die Frau an die Front, und man stößt auf einen rigorosen weiblichen Patriotismus, der jede Unterwerfung oder auch nur Verständigung radikal ablehnt. Die Frau erscheint in einer Vermengung von Konkretem und Symbolischem in der entstandenen nationalen Notlage als bewaffnete Streiterin neben und hinter dem Mann, um den Kampf auch nach dessen Tod, dann vermutlich noch kompromiß- und schonungsloser, fortzusetzen. Auch die französische Propaganda lebte vom Rekurs auf Weibliches, wie Victor Hugos Ansprache an die Franzosen vom 17. September belegt. „Verteidigung bis zum Äußersten“ fordernd, rief er seinen Landsleuten zu:

Jeder sei ein Camille Desmoulin, jede Fran eine Théroigne, jeder Jüngling ein Barra. [...] Rollet Felsen herab, häufet Pflastersteine, verwandelt die Pflugschare in Beile, verwandelt die Furchen in Gräben, kämpft mit allem, was euch in die Hand fällt, nehmt die Steine unseres geheiligten Bodens, steinigt die Eindringlinge mit den Gebeinen unserer Mutter Frankreich. ${ }^{\text {It }}$

Exzesse blieben nicht aus: So kam es in der bereits erwähnten Ortschaft Bazeilles bei Sedan, der Exkurs sei erlaubt, zu Greueln, als sich Einwohner zur Wehr setzten, darunter eine verzweifelte Mutter, die ihre Söhne im Krieg verloren hatte. Sie tötete vordringende bayerische Soldaten mit einem Jagdgewehr und wurde daraufhin mit weiteren Zivilisten von den Deutschen niedergemacht. Als „Furie von Bazeilles“ in die deutsche Propaganda eingegangen, hieß es dort in einer noch moderaten Version, daß an den Vorgängen „Weiber mit gellendem Gelächter wie Hyänen“ beteiligt waren, „Megären mit rauchender Flinte und blutigem Säbel“"52. Auch um Metz sollen alte Weiber "gleich Hexen auf dem Schlachtfelde“ ihr Unwesen getrieben haben, wovon man eines aufhängen ließ, „das dabei betroffen wurde, wie es einem ver-

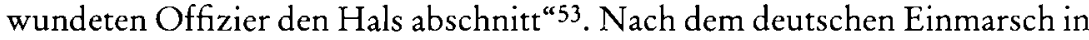
der Stadt kam es dann aber offensichtlich zu keinerlei Aufbegehren. Nur zeigten sich einige Frauen in schwarzen Kleidern, und auch später fand man noch häufig Bäuerinnen in Schwarz, die um ihr Vaterland trauerten ${ }^{54}$. Die nach Kriegsende in den Denkmälern entlang der Grenze zu den abgetretenen Gebieten verewigten Frauengestalten ${ }^{55}$ spiegelten diese Trauer, aber auch den

50 Aufruf der Arbeiterin Hestair im L'Indépendant de la Moselle vom 16. Oktober 1870. Zit. nach: WestPhal, Metz, Teil III, S. $316 \mathrm{f}$.

51 Zit. nach HIRTH, Tagebuch, Bd. II, S. 2244.

52 BleibTReu, Sedan, S. $96 \mathrm{f}$.

53 Kretschman, Kriegsbriefe, S. 80.

54 Ibid S. 230.

55 Vgl. MAAs, Kriegerdenkmäler, S. 97. 
Willen, Revanche zu nehmen und das verlorene Land zurückzuerobern, und sie standen so durchaus in der Tradition des während der Belagerung aufgekommenen weiblichen Patriotismus.

Auch das Problem sexueller Abstinenz, noch nicht, wie in späteren Kriegen, durch ein organisiertes Bordellwesen aufgewogen, berührt das Thema Frauen. Zu Kontakten mit Prostituierten kam es deutscherseits insgesamt noch selten, was aber offenbar weniger auf mangelnde Gelegenheit als eine durch die Kirche vermittelte Prüderie in allen sexuellen Belangen zurückzuführen war ${ }^{56}$.Zudem fehlte pornographisches Bildmaterial, das erst die kommerzielle Erotik der wilhelminischen Zeit hervorbringen sollte. Vor Metz indes fanden sich mit der Zeit Damen des horizontalen Gewerbes ein, und selbst unter der genannten mentalen Konstitution des Durchschnittssoldaten war es auf Dauer unmöglich, davor Augen und Ohren zu verschließen. Man fing also an, Französinnen kennenzulernen, von denen man, und allein dieses spricht Bände, bislang geglaubt hatte, es gäbe sie nur in Paris:

Französische Frauenzimmer, die sich sämtlich für die Frauen von Offizieren ausgeben, treiben sich in elegantester Toilette und mit den nie täuschenden Merkmalen ihrer sozialen Stellung überall umher. Es werden natürlich Vorkehrungen getroffen, diesem Treiben ein Ende zu machen. ${ }^{57}$

Eine gewisse Mischung von Antifeminismus und Franzosenfeindlichkeit prägt hier ganz offensichtlich die Abwehrhaltung nicht nur gegen einen moralisch verwerflichen Frauentyp, sondern jedweden weiblichen Einfluß auf Krieg und Militär. Auch wird die vermeintliche Dekadenz Frankreichs auf eine entartete Geschlechterordnung zurückgeführt: Äußerlich kraftvollere und zugleich willensstärkere Frauen dominierten dort über ihre „dünnbeinigen, zappligen Männer", und es sei gar nicht rätselhaft, daß sich in diesem Lande alles um die Frau drehe - ein Zug, der untergehenden Nationen überhaupt anhafte ${ }^{58}$. Als der zur sittlichen Wehrhaftigkeit passende Frauentyp erscheint hingegen jener, der, gewissermaßen von Ferne, "Gesinnungen der Ehre, des Pflichtgefühls, der Opferfähigkeit“ zu erzeugen vermag59 gleichsam der Gegenentwurf zu französischer, eben unsittlicher Weiblichkeit, wie man ihr auch vor Metz zu begegnen glaubte. Vor allem nach dem Einzug in die Stadt, wo sich "liederliche Frauenzimmer" herausgeputzt und den Regen benutzt hatten, „ihre Röcke unter dem Arm zu tragen“60, versuchte die militärische Führung, Kontakte mit städtischen Huren zu unterbinden, von denen nach einer im Dezember durchgeführten Untersuchung mehr als die Hälfte geschlechtskrank war ${ }^{61}$. Doch war dies, wie angedeutet,

$56 \mathrm{KÜHLICH}$, Soldaten, S. $292 \mathrm{f}$.

57 KRETSCHMAN, Kriegsbriefe, S. 119.

58 Ibid S. 352.

59 Ibid.

60 Ibid S. 190.

61 Den Statistiken zufolge waren in der Stadt von den offiziell zugelassenen Prostituierten zwar nur $6 \%$, von den inoffiziell anschaffenden Damen hingegen schon $66 \%$ ge- 
nur schwer durchzusetzen. Sinnfällig wurde hier, wie nach dem Einzug deutscher Truppen in Paris Anfang März 1871, daß gerade längere Aufenthalte in größeren Orten Tabus aufweichten. Überdies verfestigte sich das auch in Unterschichten gängige Bild von den leichtlebigen Französinnen, das mit der Herausstellung sittenloser und sündhafter Zustände insbesondere in der französischen Hauptstadt korrespondierte und, wie oben exemplifiziert, überhaupt als ein Zeichen nationaler Dekadenz galt ${ }^{62}$. Frankreich, so konstruierte man, war verweiblicht und damit moralisch verkommen. Diese Vorstellung wurde zum Topos im Denkhaushalt des Kaiserreichs, und noch mein Urgroßvater schrieb 1929 in einem Gedicht über den Ersten Weltkrieg: "Nu hattn $\mathrm{mr}$ enn Feldwebel,/ dos war e dicker Wanst./ Mit mancher schenn Franzusndam'/ hot dar in Krieg getanzt.// Auch die den Franzosen unterstellte Lust zum Krieg führte man so nicht nur auf nationalen Ehrgeiz zurück, sondern brachte sie mit dem weiblich konnotierten Charakterzug der Frivolität in Zusammenhang ${ }^{63}$. Die Belles Françaises aber vermißte beim Abmarsch von Metz ins Landesinnere auch der preußische Offizier: „Sie verstecken sich vor uns, denn nur eine Kategorie alter Weiber ist sichtbar, die man dreist ohne Maske bei jedem Hexensabbat figurieren lassen kann." 64

Gegenüber den oben erwähnten verdächtigen „Frauenzimmern“ liest sich die Geschichte einer Französin, die in der Gartenlaube unter dem Titel Laurence erschien, wie eine Parodie: Laurence bittet im Hauptquartier um Einlaß nach Metz, wo sich ihr verwundeter Mann befindet. "Eine Dame“, so orakelt der Berichterstatter dazu, „die sich in den Mittelpunkt des Krieges wagt, besitzt Muth und Entschlossenheit, und eine Frau mit diesen Eigenschaften kann nicht zu den Gewöhnlichen ihres Geschlechts gehören "65. Im Laufe der Erzählung zeigen sich dann aber doch die „gewöhnlichen Schwächen" des Weibes, etwa, wenn es nicht weiß, daß Schlesien zu Preußen gehört: „Wir Frauen in Frankreich“, so erklärt Laurence ihre und ihrer Landsmänninnen Schwäche gleich mit, „treiben Geographie nur mit unserem Herzen"66. Von zwei Nonnen wird die „anständige, wohlgekleidete“ Dame, hierin offenbar die Ausnahme unter ansonsten entweder "liederlichen“ oder eben "häßlichen Frauenzimmern“, nachdem ihr die rührigen Preußen zumindest ein sicheres Lebenszeichen des Geliebten vermittelt haben, schließlich nach Belgien, also auf neutralen Boden, begleitet.

Liebschaften und engere Bindungen deutscher Soldaten mit Französinnen,

schlechtskrank. OMPTEDA, Krankenpflege, S. 409. Es muß also auch eine nicht unbeträchtliche, vermutlich stetig steigende Zahl geschlechtskranker Soldaten gegeben haben, die von der Statistik jedoch noch nicht erfaßt wurde.

62 KüHLICH, Soldaten, S. $135 \mathrm{f}$.

63 Kretschman, Kriegsbriefe, S. 223.

64 Ibid S. 196.

65 Gartenlaube (1870), Nr. 47, S. 776.

66 Ibid S. 778. 
wie sie Remarques Paul Bäumer im Ersten Weltkrieg erlebte, waren nicht gänzlich ausgeschlossen, blieben aber doch Seltenheit. Zumindest galt das für die Anfangsphase des Krieges. Später scheint es Verhältnisse vor allem zwischen höheren Offizieren und Damen der französischen Oberschicht gegeben zu haben. Als die Deutschen am 9. März 1871 Le Mans räumten, erkundigten sich „Frauen aus den besseren, selbst besten Ständen" nach Offizieren, „qui avaient promis de revenir encore une fois. Selbst Tränen flossen dabei“"67. Insgesamt zeichnete sich ab, daß Soldatentum und Sexualität künftig kein Gegenstand moralisierender Bewertung mehr sein konnte, sondern eine Frage von praktischer Bedeutung. Zur Kanalisierung überschüssiger Energien und zur körperlichen Ertüchtigung ließen einige Kompanieführer vor Metz neben dem Exerziertraining auch turnen ${ }^{68}$. Interessant in diesem $\mathrm{Zu}$ sammenhang ist schließlich noch, daß nüchterne französische Stimmen gerade das respektvolle Verhalten der Deutschen gegenüber den einheimischen Frauen herausstellten und sogar für „une qualité nationale, et une des sources de la force de la race germanique" hielten ${ }^{69}$.

\section{Kleinkrieg und Beziehungen zwischen Deutschen und Franzosen}

Abgerechnet der Ausfälle, bestand die französische Kriegsführung darin, die deutschen Vorposten durch Artilleriebeschuß aus den Festungsgeschützen und gelegentliche Infanterieüberfälle in Atem zu halten. Dieser Kleinkrieg sollte die Belagerer zermürben und die eigene Offensivkraft stärken. Überfälle und Scharmützel im Vorpostenbereich wurden mit Fortdauer der Belagerung von beiden Seiten inszeniert und als abenteuerliche Abwechslung des monotonen Wachdienstes empfunden ${ }^{70}$ : Patrouillen schleichen im Morgengrauen in den feindlichen Postenbereich, überfallen die gegnerischen Wachen und ziehen sich rasch wieder zurück. Waffen und Verpflegung werden dabei erbeutet, manchmal auch Pferde. Nicht selten gibt es Tote und Verwundete ${ }^{71}$. Gefangene sollen anfangs noch durchaus gemacht werden, auch um zu wissen, wie es beim Gegner steht. Wo gelungen, wird dies immer als eine Art Heldentat zum Anlaß abenteuerlichster Erzählungen. Es kommt vor, daß man eigene Leute in französische Uniformen steckt, um sich hervorzutun. So

67 KRETSCHMAN, Kriegsbriefe, S. 358.

68 SCHREIBER, Geschichte des Infanterie-Regiments, S. 298.

69 MONOD, Allemands et Francais, S. $65 \mathrm{f}$.

70 HaAs, Kriegs-Erinnerungen, S. 46. TifdemanN, Erinnerungen, S. 26f. KRETSCHMAN, Kriegsbriefe, S. 164. STEINBECK, Um und in Metz, S. 119 und 127.

71 GOLTZ, Feldzug, S. 287. Weitaus gefährlicher waren derartige Unternehmen im Ersten Weltkrieg, wo die Stoßtrupps mit Handgranaten und Flammenwerfern vorgingen. Vgl. JÜNGER, In Stahlgewittern, S. $98 \mathrm{f}$. 
wird eines Tages ein vermeintlich gefangener Garde-Voltigeur ${ }^{72}$ dem Prinzen Friedrich Karl zum Verhör vorgeführt. Als dieser ihn ausgefragt und minutenlang französisch auf ihn eingesprochen hat, hält es jener schließlich nicht mehr aus und sagt: „Ach lassen sie mir doch zufrieden, ick bin ja man bloß een 35 er"73.

Mit ihren weitreichenden Chassepots hielten die französischen Vorposten die deutschen Wachen in Atem. Es wurde viel, oft ungezielt und "wild“ geschossen, manchmal bloß, um die eigene Angst zu vertreiben. Nach und nach hatten auch die Einschließungstruppen genügend Beutewaffen, um sich zu revanchieren. So kam es zu ständigen Plänkeleien zwischen den Vorposten mit Verlusten auf beiden Seiten ${ }^{74}$. Die sich zuspitzende Hungerlage in der Stadt zwang die Franzosen, kleine Ausfälle mehr und mehr in den Dienst der Fouragierung, also der Beschaffung von Lebensmitteln für Militär und Zivilbevölkerung, zu stellen. In diesen Auseinandersetzungen gingen die Ortschaften und Gehöfte um Metz nach und nach in Flammen auf. Sie wurden in Brand geschossen oder durch die jeweiligen Besitzer selbst - die Deutschen praktizierten dies, um französischen Absichten zuvorzukommen - geräumt und zerstört. Leidtragende waren die Zivilisten, die häufig bis zuletzt in ihren Häusern ausharrten:

Die ganze Nacht leuchtete der Feuerschein, und heute früh kam die arme Bevölkerung, die man nachts aus den Betten geholt hat und nun einige Stunden weiter rückwärts einquartieren wird, mit dem eilig zusammengerafften bischen Habe hier durch! Ein jämmerlicher Zug. Hier und da ein mühsam durch alle Anfechtungen durchgefristetes Pferd mit Betten bepackt, oder mit einem Kranken darauf, Weiber mit Säuglingen an der Brust und einem hochgethürmten Korb voll Hausrath auf dem Rücken, Kinder, die kaum laufen konnten, und halb stumpfsinnige Alte, Alles mit bitterer Verzwciflung im Gesicht [...], voll finster drohender Minen. ${ }^{75}$

Die einst wohlhabende Gegend mit ihren schönen Bauernhöfen und im italienischen Renaissancestil errichteten Villen und Schlössern sank in Schutt und Asche. Menschen, die eben noch unter günstigen Bedingungen gelebt und gearbeitet hatten, sahen sich ihrer Lebensgrundlage beraubt und standen vor den Trümmern jahrelangen Schaffens. Womöglich hatten sie noch einen Angehörigen bei Sedan oder Metz verloren. Überhaupt griff der Krieg um die Festung bereits stark in den zivilen Bereich aus, und es schien zur Normalität zu gehören, daß Unbeteiligte in die Feuerzonen gerieten:

Du machst Dir keinen Begriff, welch traurigen Eindruck die Ortschaften um Metz machen. Alle Häuser mehr oder minder durch Granatfeuer demoliert; viele ganz niedergebrannt; alle Dächer eingeschlagen; noch die Reste zeugen von großer Wohlhabenheit der Besitzer. Die ärmeren Leute blieben zurück. Unglaubliche Gestalten, alte Weiber mit Herrenphysiognomien huschen scheu an den Mauern entlang, Männer mit verhun-

72 LÄUYER, Ordonnanz.

73 KRETSCHMAN, Kriegsbriefe, S. 110 f.

74 Ibid S. 98.

75 RinifleisCH, Feldbriefe, S. 49. Das erinnert stark an Bilder, wie man sie aus dem Kosovo oder aus Tschetschenien noch gut in Erinnerung hat. 
gerten Zügen, auf denen die innere Wut noch einen Platz behielt, stehen an den Trümmern und fassen hin und wieder nach dem Munde, als ob sie die gewohnte Pfeife ergreifen wollten, die sie sich seit Wochen versagen müssen. ${ }^{76}$

Kehren wir noch einmal zu den Vorposten zurück. Dort, wo das Gehölz in einem breiten Gürtel verschwunden und Chausseebäume abgehackt waren, um dem Gegner die Deckung zu nehmen und der Artillerie freie Schußbahn zu verschaffen, hatte man vor dem Krieg Kartoffeln angebaut. Jetzt lagen die zertretenen Felder zwischen den feindlichen Linien, und die nahrhaften Knollenpflanzen gehörten wie die Äcker, in denen sie steckten, niemandem. Bald begannen die französischen Vorposten, die Feldfrüchte im Niemandsland aufzusammeln. Anfangs entwickelten sich daraus noch kleinere Schießereien; später, als die schlechte Ernährungslage in Metz bei den Belagerern bekannt wurde, gestatteten die deutschen Vorposten den halb verhungerten Franzosen stillschweigend das Suchen. Man denkt an Friedrich den Großen, der seine Kartoffelfelder einst bewachen ließ, um die neu eingeführten Kulturen vor religiösen Fanatikern zu schützen. Auf Vorposten verständigte man sich bei solchen Unternehmen per Zeichensprache:

Es dauerte nicht lange, da nahmen die Franzosen ihre Käppis ab und grüßten herüber, von hier aus erfolgte Gegengruß. Nach einiger Zeit steckten die Franzosen den Daumen der rechten Hand in den Mund und schlenkerten den Arm nach unten. Dies war allgemein in Frankreich bei den Bauern üblich, wenn man sie fragte, ob sie was zum Essen im Hause hätten, dann machten sie diese Bewegung mit dem Daumen. Nachdem im allgemeinen die Freundschaft hergestellt war, schwenkte einer der Franzosen das Taschentuch und zeigte auf das vorliegende Terrain, woselbst sich ein Kartoffelstück befand. Die Unsrigen nickten zu. Blitzschnell sprang einer aus seinem Loche und nach dem Kartoffelstück, woselbst er in aller Eile seinen Brotbeutel oder das Taschentuch voll Kartoffel ausbuddelte und wieder in seine Stellung verschwand. Dann lüfteten sie zum Zeichen des Dankes wieder ihre Käppis. ${ }^{77}$

Dabei war es gut möglich, daß Wachen, die eben noch miteinander gesprochen und Lebensmittel getauscht hatten, im nächsten Augenblick wieder aufeinander schossen. Immer häufiger zeigten sich aber französische Posten unbewaffnet, und schließlich führten sie sogar Zivilisten mit auf die Felder. Da dieser Modus vivendi nicht der Aushungerungstaktik der deutschen Führung entsprach, befahl der Oberkommandierende der Truppen vor Metz, Prinz Friedrich Karl, am 21. September, „daß der Vorpostendienst in aller Schärfe betrieben werde und daß Unterlassungen - die, auf beiderseitigem stillschweigenden Einverständnis beruhend, in früheren Kriegen bei andauernden Vorpostenaufstellungen vorgekommen sind -, nicht geduldet wer-

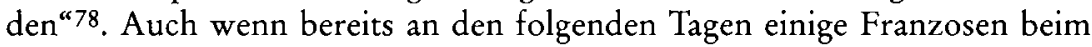

76 KRETSCHMAN, Kriegsbriefe, S. 108.

77 HaAs, Kriegs-Erinnerungen, S. $51 \mathrm{f}$.

$78 \mathrm{Im}$ Oktober häuften sich Verbrüderungsszenen im Vorpostenbereich. Es bestand die Gefahr, daß ganz im Sinne Bazaines ein Teil der Rheinarmee die Waffen strecken und der andere Teil die Festung weiter besetzt halten könnte. Daher der entsprechende Be- 


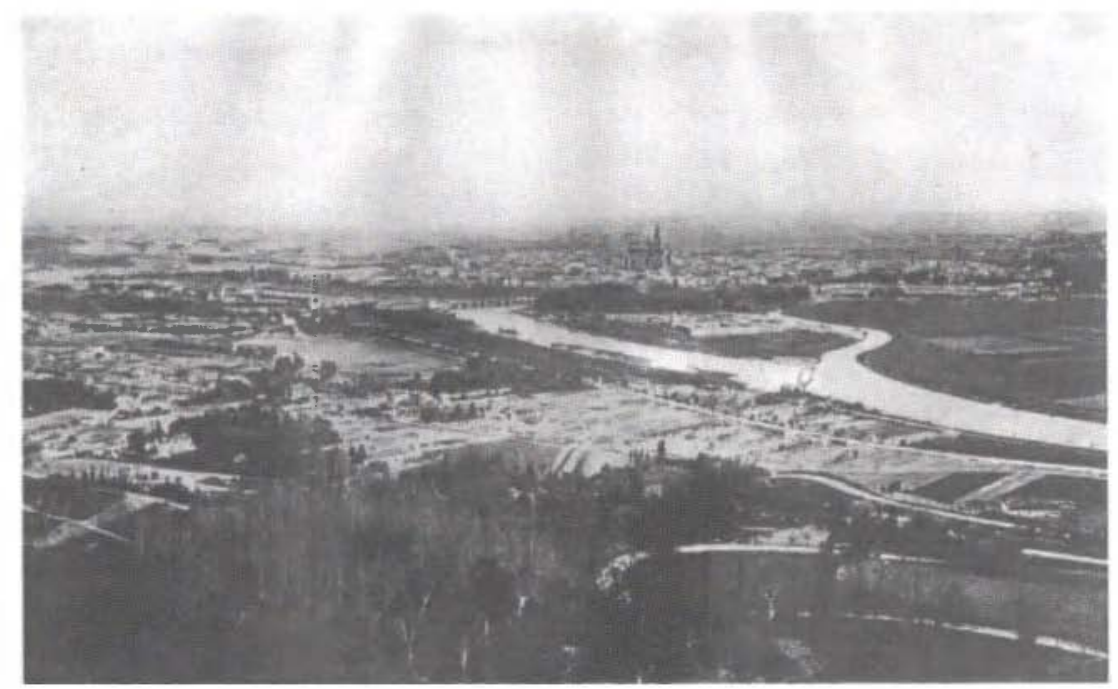

Abb. 13: Blick auf Metz von Südwesten. Quelle: TıEDIMANN, Erinnerungen.

Kartoffelsuchen erschossen wurden ${ }^{79}$, brachen die inoffiziellen, spontan entstandenen Beziehungen nicht ab. So nutzten die Franzosen jede Waffenruhe, um bei den Deutschen um Brot, Salz und Schnaps zu bitten. Später trieb sie der Hunger in Massen zu den deutschen Linien, um sich gefangen zu geben. $\mathrm{Da}$ die Deutschen jedoch Befehl hatten, Überläufer abzuweisen, solange Metz nicht kapitulierte, wurden diese mit ungezieltem Feuer, am Schluß sogar mit Knüppeln und Gewehrkolben zurückgetrieben ${ }^{80}$. Regelmäßig wechselten Parlamentäre die Seiten, um den Austausch Gefangener vorzubereiten oder eine befristete Waffenruhe zur Bergung Toter und Verwundeter zu vereinbaren. Andere schleuste man in geheimer Mission durch die Linien, so den Kommandeur der kaiserlichen Garde, General Bourbaki, der Ende September incognito mit einer Gruppe Rot-Kreuz-Ärzte zu separaten Friedensverhandlungen in Richtung England aus der Festung gebracht worden war ${ }^{81}$. Diese geheimen Kontakte zur Rheinarmee hatte Bismarck im übrigen ganz selbständig ins Werk gesetzt; die militärische Führung vor Metz blieb davon ohne nähere Kenntnis. Dort witterte man Gefahr, den zu erwartenden militärischen Erfolg durch politische Verhandlungen zu verlieren ${ }^{82}$. So weigerte

fehl. Vgl. Goltz, Feldzug, S. 283 f. Auch die französische Führung versuchte, dagegen einzuschreiten. Vgl. FAY, Tagebuch, S. 183.

${ }^{79}$ Ibid.

80 Goltz, Feldzug, S. 389 f. TiedemanN, Erinnerungen, S. $37 \mathrm{f}$.

$81 \mathrm{Vgl}$. KolB, Weg aus dem Krieg, S. 260. FAY, Tagebuch, S. $140 \mathrm{f}$.

82 Im Fall Bourbaki glaubte man lange an ein Entweichen durch die stillgelegte Wasserleitung, die von Gorze nach Metz führte. 
sich Prinz Friedrich Karl trotz königlichen Befehls, Bourbaki nach dessen gescheiterter England-Mission wieder nach Metz hinein zu lassen. ${ }^{83}$

$\mathrm{Zu}$ friedlichen Kontakten zwischen den Belagerern und der französischen Zivilbevölkerung kam es trotz allem. Obgleich sich die Masse vornehmlich der begüterten, durch den Weinanbau reich gewordenen Bewohner nach Metz oder ins Landesinnere geflüchtet hatte, blieben doch eine Reihe meist ärmerer, bodenständiger älterer Leute in ihren Häusern ${ }^{84}$. Wenn es nicht, wie schon erwähnt, zur Vertreibung aus militärstrategischen oder aus Gründen der Bestrafung kam, so konnten mit der Zeit durchaus engere Beziehungen zwischen Wirtsleuten und einquartierten oder in der Nähe lagernden Soldaten entstehen. Man gewöhnte sich an ein Leben miteinander und arrangierte sich, soweit möglich, zum beiderseitigen Vorteil:

Ich lag mit verschiedenen Kameraden bei einem alten Ehepaar von zirka 70 Jahren, das nicht geflüchtet war. Der Mann war Tagelöhner und arbeitete im Weinberge. Bei ihrer Armut und Einfachheit waren es biedere und zufriedene Menschen. Sie hatten ein kleines Häuschen am Rande des Dorfes mit einem Raume von zirka 25 Quadratmeter Flächeninhalt. Dieser Raum war Wohnzimmer, Schlafzimmer und Küche. Der Fußboden bestand aus Lehmschlag. Das ganze Mobiliar bestand aus einem Bette, um welches ein rotkarrierter Vorhang angebracht war, einem derben Tische und mehreren Stühlen, ferner einer großen Kiste (Truhe), einer Bank und einem gemauerten, offenen Herdfeuer. Neben dem Häuschen war ein Schuppen mit Ziegenstall angebaut [...]. Wir schliefen ein Teil im Ziegenstall, der andere Teil, unter welchem auch ich mich befand, auf dem Futterboden über dem Ziegenstall [...]. Um die alten Leute, welche sehr gefällig waren, so wenig wie möglich in ihrer Häuslichkeit zu stören, kampierten wir immer außerhalb des Häuschens ${ }^{85}$. Wir schleppten nun Proviant herbei. Die alte Frau war uns behilflich beim Kochen, der alte Mann ging mit einem oder zwei von uns auf Holzsuche; er wußte immer, wo was zu finden war [..... Unser Quartierwirt und seine Frau waren während des Aufenthaltes im Dorf natürlich unsere Gäste. ${ }^{86}$

Im Metzer Umland sprach man jedoch kaum deutsch, so daß Soldaten und Einheimische nur selten näher ins Gespräch kamen. Über ausreichende Französischkenntnisse verfügte nur ein kleiner Teil der Offiziere, vom einfachen Mann ganz zu schweigen. Doch gerade auf die Verständigung kam es nun einmal an, wenn man nicht nur nehmen, sondern auch verhandeln und übereinkommen wollte:

Dann bin ich merkwürdigerweise derjenige im Hauptquartier, der sich am schnellsten verständigt: mit meinem Französisch! Aber enfin, die Bewohner behaupten, sie verständen mich besser. Mit $61 / 2$ Vokabeln kann man eine Menge sagen; ich glaube aber, daß ich schon bis auf 10 gekommen bin. Übrigens reden die Leute hier ein verteufeltes Französisch. ${ }^{87}$

${ }^{83} \mathrm{Vgl}$. Kolk, Weg aus dem Krieg, S. 260. Bourbaki stellte sich daraufhin der provisorischen Regierung zur Verfügung und crhielt später das Kommando über die französische Ostarmee, die er nach Kriegsende auf neutralen Schweizer Boden führte.

84 HAAS, Kriegserinnerungen, S. 27f. KRETSCHMAN, Kriegsbriefe, S. 94 und 108.

85 Es war während der sonnigen Tage zwischen dem 11. und 24. September 1870.

${ }^{86}$ HaAS, Kriegs-Erinnerungen, S. 29. Ähnlich auch: KoCH, Bei den Fahnen, S. $17 \mathrm{f}$.

87 KRETSCHMAN, Kriegsbriefe, S. 86. Zur Französischschwäche trotz mehrjähriger 
Die Bevölkerung empfand es daher auch als ungewöhnlich, wenn ein Deutscher französisch sprach. Vom Einzug in Metz berichtete ein preußischer Leutnant verwundert, daß sich immer, wenn er mit Einheimischen französisch zu sprechen begann, unter freudigen Rufen ,il parle, il parle!“ sofort ein großer Kreis um ihn bildete ${ }^{88}$. Natürlich hing dies auch mit dem Informationshunger der Eingeschlossenen zusammen. In elementaren Fragen aber funktionierte die Verständigung mit Händen und Füßen:

So erzählte man, ein Bayer hätte erklärt, er verstände schon ganz gut Französisch, um sich mit den Wirtsleuten zu unterhalten; und näher befragt, gab er an, er zöge sofort seine Uhr heraus, deutete an vier Stellen auf dieselbe, $4 \mathrm{mal}$ "manger" sagend und umschreibe dann mit dem Zeigefinger den ganzen Radius des Zifferblattes mit den Worten „toujours boire"; dann wüßten die Leute, daß er am Tage 4 mal essen und fortwährend trinken wolle. ${ }^{89}$

Wie wir hörten, konnte ein solches Programm vor Metz nur in Ausnahmefällen realisiert werden. Dennoch war, so lassen die Quellen erkennen, im Schatten der überwiegenden Härten des Belagerungsalltags ein einvernehmliches Verhältnis zwischen Besatzern und einheimischen Bauern durchaus möglich. Solche Beziehungen konnten sogar zum Abbau von Vorurteilen und Feindbildern führen: So merkte der deutsche Soldat, daß Franzosen eben nicht nur „verkommene Subjekte“, sondern auch umgängliche Menschen sein konnten, und der französische Bauer sah, daß sich die „Barbaren aus dem Osten " ihm gegenüber durchaus korrekt zu verhalten wußten ${ }^{90}$. Derartiges senkte sich aber kaum tiefer in die Hirne der Beteiligten ein, und Stimmen, die schon damals den Unterschied zwischen nationaler Propaganda und den wirklichen Zuständen offenzulegen suchten ${ }^{91}$, wurden von einer heroischen Kampferfahrung verdrängt, die dem Feind all diejenigen Defizite zuschrieb, deren es bedurfte, um ihn als solchen zu begreifen.

Schulausbildung bei den Deutschen vgl. u. a.: HAAS, Kriegs-Erinnerungen, S. 27. KÜHLICH, Soldaten, S. $97 \mathrm{f}$.

${ }^{88}$ Brief eines preußischen Offiziers aus Metz, in: WZ, Jg. 1870, Nr. 263.

89 BRONSART, Kriegstagebuch, S. 147. Der Kenner weiß, daß die Bayern nicht vor Metz lagen. Die Geschichte soll sich vor Paris ereignet haben und wurde zur Erheiterung der Geburtstagfeier des Grafen von Moltke am 26. Oktober in Versailles erzählt. Dennoch darf derartiges „Besatzerlatein“ cum grano salis stellvertretend für vergleichbare, vor Metz stattgefundene Verständigungen erwähnt werden.

$90 \mathrm{KoCH}$, Bei den Fahnen, S. 39. Wie sich das Frankreichbild deutscher Soldaten von 1870 über den Ersten bis zum Zweiten Weltkriege wandelte, wäre einmal gesondert an Hand von Feldpostbriefen zu untersuchen.

91 Dafür auf französischer Seite: GOBINEAU, Ce qui est arrivé. 
